\title{
Approximating Shepp's constants for the Slepian process
}

\author{
Jack Noonan ${ }^{\mathrm{a}}$, Anatoly Zhigljavsky, ${ }^{\mathrm{a}, *}$ \\ ${ }^{a}$ School of Mathematics, Cardiff University, Cardiff, CF24 4AG, UK
}

\begin{abstract}
Slepian process $S(t)$ is a stationary Gaussian process with zero mean and covariance $\mathbb{E} S(t) S\left(t^{\prime}\right)=$ $\max \left\{0,1-\left|t-t^{\prime}\right|\right\}$. For any $T \geq 0$ and real $h$, define $F_{T}(h)=\operatorname{Pr}\left\{\max _{t \in[0, T]} S(t)<h\right\}$ and the constants $\Lambda(h)=-\lim _{T \rightarrow \infty} \frac{1}{T} \log F_{T}(h)$ and $\lambda(h)=\exp \{-\Lambda(h)\}$; we will call them 'Shepp's constants'. The aim of the paper is construction of accurate approximations for $F_{T}(h)$ and hence for the Shepp's constants. We demonstrate that at least some of the approximations are extremely accurate.
\end{abstract}

Keywords: Slepian process, extreme value theory, boundary crossing probability

\section{Introduction}

Let $S(t), t \in[0, T]$, be a Gaussian process with mean 0 and covariance

$$
\mathbb{E} S(t) S\left(t^{\prime}\right)=\max \left\{0,1-\left|t-t^{\prime}\right|\right\}
$$

This process is often called Slepian process. For any real $h$ and $x<h$, define

$$
F_{T}(h \mid x):=\operatorname{Pr}\left\{\max _{t \in[0, T]} S(t)<h \mid S(0)=x\right\} ;
$$

if $x \geq h$ we set $F_{T}(h \mid x)=0$. Assuming that $x$ has Gaussian distribution $N(0,1)$, and hence the stationarity of the process $S(t)$, we average $F_{T}(h \mid x)$ and thus define

$$
F_{T}(h):=\int_{-\infty}^{h} F_{T}(h \mid x) \varphi(x) d x,
$$

where $\varphi(x)=(2 \pi)^{-1 / 2} \exp \left\{-x^{2} / 2\right\}$.

Key results on the boundary crossing probabilities for the Slepian process have been established by L.Shepp in [1]. In particular, Shepp has derived an explicit formula for $F_{T}(h)$ with $T$ integer, see 2.5 below. As this explicit formula is quite complicated, in (3.7) in the same paper, Shepp has conjectured the existence of the following constant (depending on $h$ )

$$
\Lambda(h)=-\lim _{T \rightarrow \infty} \frac{1}{T} \log F_{T}(h)
$$

and raised the question of constructing accurate approximations and bounds for this constant.

The importance of this constant is related to the asymptotic relation

$$
F_{T}(h) \simeq \operatorname{const}[\lambda(h)]^{T} \text { as } T \rightarrow \infty
$$

where $\lambda(h)=\exp \{-\Lambda(h)\}$. We will call $\Lambda(h)$ and $\lambda(h)$ 'Shepp's constants'.

\footnotetext{
${ }^{*}$ Corresponding author

Email addresses: NoonanJ1@cardiff.ac.uk (Jack Noonan), ZhigljavskyAA@cardiff.ac.uk (Anatoly Zhigljavsky)
} 
In this paper, we are interested in deriving approximations for $F_{T}(h)$ in the form 1.5 and hence for the Shepp's constants. In formulation of approximations, we offer approximations for $F_{T}(h)$ for all $T>2$ and hence approximations for $\Lambda(h)$ and $\lambda(h)$. Note that computation of $F_{T}(h)$ for $T \leq 2$ is a relatively easy problem, see [2] for $T \leq 1$ and 1 for $1<T \leq 2$.

In Section 2 we derive several approximations for $F_{T}(h)$ and $\lambda(h)$ and provide numerical results showing that at least some of the derived approximation are extremely accurate. In Section 3 we compare the upper tail asymptotics for the Slepian process and some other stationary Gaussian processes. Section 4.1 contains some minor technical details and Section 5 delivers conclusions.

\section{Construction of approximations}

\subsection{Existence of Shepp's constants and the approximations derived from general principles}

The fact that the limit in 1.4 exists and hence that $\Lambda(h)$ is properly defined for any has been proven in 3. The proof of existence of $\Lambda(h)$ is based on the inequalities

$$
-\frac{1}{n+1} \log \left[F_{n}(h)\right] \leq \Lambda(h) \leq-\frac{1}{n} \log \left[F_{n}(h)\right] \text { for any } n=1,2, \ldots
$$

The inequality in the rhs of (2.1) follows directly from the infamous 'Slepian inequality' established in 4]; this inequality holds for any Gaussian stationary process with non-negative correlation function. The inequality in the lhs of (2.1) can be obtained by a simple extension of the arguments in [4, p.470]; it holds for any Gaussian stationary process which correlation function vanishes outside the interval $[-1,1]$. The inequalities (2.1) are not sharp: in particular, for $n=2$ and $h=0$, (2.1) gives $1.336<\Lambda(0)<2.004$; see [5, Remark 3]. As follows from Tables 1 and 3, an accurate approximation for $\Lambda(0)$ is $\Lambda(0) \simeq 1.5972$, where we claim all four decimal places are accurate.

If $n$ is not too small, the bounds (2.1) are very difficult to compute. For small $h$, these bounds are not sharp even if $n$ is large, see Fig. $1 \mathrm{a}$. The bounds improve as $h$ grows, see Fig. $1 \mathrm{~b}$. It is not very clear how to use these bounds for construction of accurate approximations for $\Lambda(h)$. In particular, from Fig. $1 \mathrm{~b}$ we observe that the upper bound of 2.1 can be much closer to the true $\Lambda(h)$ than the lower bound.

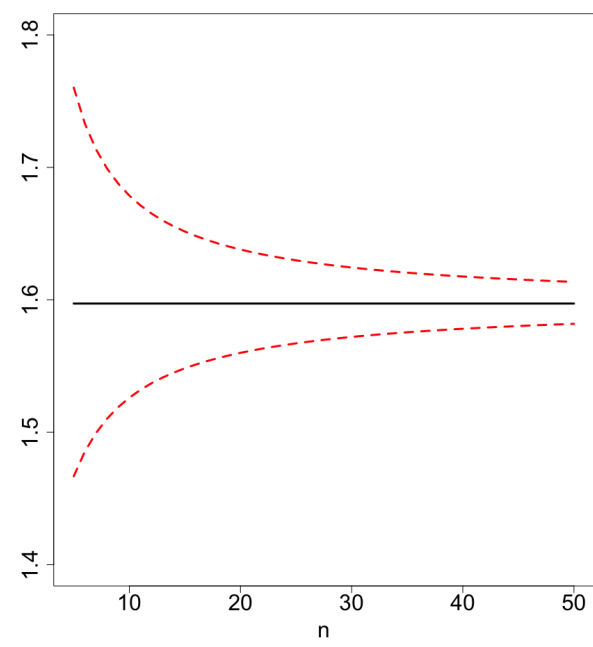

(a) $h=0$

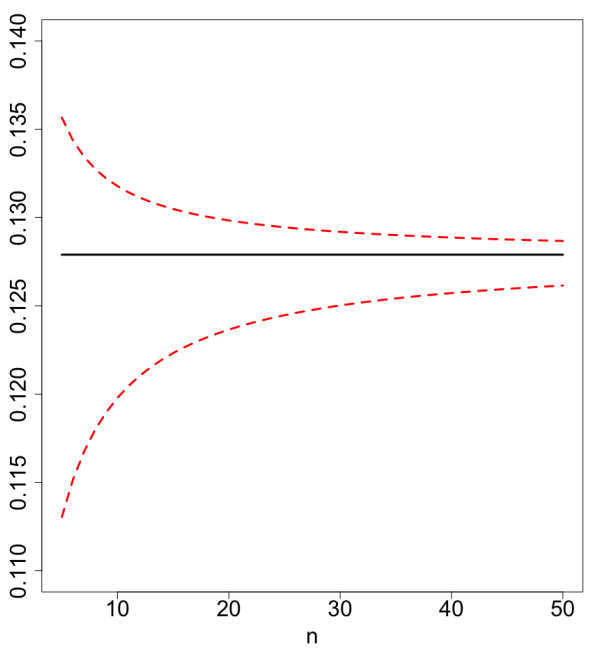

(b) $h=2$

Figure 1: Lower and upper bounds 2.1 (red dotted lines) for $\Lambda(h)$ (solid black line).

One may apply general results shown in [6, 7], see also formula (2.1.3) in [8, to approximate $F_{T}(h)$ for large $h$ but these results only show that $\lambda(h) \rightarrow 1$ as $h \rightarrow \infty$ and therefore are of no use here. A more useful tool, which can be used for approximating $\lambda(h)$, is connected to the following 
result of J.Pickands proved in [9]. Assume that $\{\xi(t)\}$ is a stationary Gaussian random process with $\mathbb{E} \xi(t)=0, \mathbb{E} \xi^{2}(t)=1$ and covariance function

$$
\rho(t)=\mathbb{E} \xi(0) \xi(t)=0=1-C|t|^{\alpha}+o\left(|t|^{\alpha}\right) \text { as } t \rightarrow 0
$$

and $\sup _{\epsilon \leq t \leq T} \rho(t)<1, \forall \epsilon>0$. Then

$$
\operatorname{Pr}\left\{\sup _{0 \leq t \leq T} \xi(t) \leq h\right\}=1-T C^{1 / \alpha} H_{\alpha} h^{2 / \alpha-1} \varphi(h)(1+o(1)) \quad \text { as } h \rightarrow \infty,
$$

where $H_{\alpha}$ is the so-called 'Pickands constant'. By replacing $1-x$ with $e^{-x}(x \rightarrow 0)$ and removing the term $(1+o(1))$ in 2.3 we obtain a general approximation

$$
\operatorname{Pr}\left\{\sup _{0 \leq t \leq T} \xi(t) \leq h\right\} \simeq \exp \left\{-T C^{1 / \alpha} H_{\alpha} h^{2 / \alpha-1} \varphi(h)\right\} .
$$

As shown in [10, the value of the Pickands constant $H_{\alpha}$ is only known for $\alpha=1,2$ and hence the approximation (2.4) can only be applied in these cases. When $\xi(t)$ is the Slepian process $S(t)$ with covariance function (1.1) we have $\alpha=1, H_{1}=1$ and $C=1$. Hence we obtain from (2.4)

Approximation 0: $F_{T}(h) \simeq \exp (-h \varphi(h) T), \quad \Lambda^{(0)}(h)=h \varphi(h), \quad \lambda^{(0)}(h)=e^{-h \varphi(h)}$.

Note that Approximation 0 can also be obtained as a Poisson clumping heuristic, see formula $(\mathrm{D} 10 \mathrm{~g})$ in [1]. If $h$ is not large, then Approximation 0 is quite poor, see Tables 1 and 2 and Figure 2. For small and moderate values of $h$, the approximations derived below in this section are much superior to Approximation 0.

\subsection{Shepp's formula for $F_{n}$}

The following formula is the result (2.15) in [1]:

$$
F_{n}(h \mid x)=\frac{1}{\varphi(x)} \int_{D_{x}} \operatorname{det}\left|\varphi\left(y_{i}-y_{j+1}+h\right)\right|_{i, j=0}^{n} d y_{2} \ldots d y_{n+1},
$$

where $T=n$ is a positive integer, $D_{x}=\left\{y_{2}, \ldots, y_{T+1} \mid h-x<y_{2}<y_{3}<\ldots<y_{n+1}\right\}, y_{0}=0, y_{1}=$ $h-x$. L.Shepp in [1] has also derived explicit formulas for $F_{T}(h \mid x)$ with non-integral $T>0$ but these formulas are more complicated and are realistically applicable only for small $T$ (say, $T \leq 3$ ).

From 2.5 we straightforwardly obtain

$$
\begin{aligned}
F_{1}(h \mid x) & =\Phi(h)-\frac{\varphi(h)}{\varphi(x)} \Phi(x), \\
F_{1}(h) & =\int_{-\infty}^{h} F_{1}(h \mid x) \varphi(x) d x=\Phi^{2}(h)-\varphi(h)[h \Phi(h)+\varphi(h)],
\end{aligned}
$$

where $\Phi(x)=\int_{-\infty}^{x} \varphi(t) d t$. Derivation of explicit formulas for $F_{T}(h \mid x)$ and $F_{T}(h)$ with $T \leq 1$ is relatively easy as the process $S(t)$ is conditionally Markovian in the interval $[0,1]$, see [12]. Formula 2.6. has been first derived in 2].

In what follows, $F_{2}(h)$ also plays a very important role. Using 2.5$)$ and changing the order of integration where suitable, $F_{2}(h)$ can be expressed through a one-dimensional integral as follows:

$$
\begin{aligned}
F_{2}(h) & =\Phi^{3}(h)+\varphi^{2}(h) \Phi(h)+\frac{\varphi^{2}(h)}{2}\left[\left(h^{2}-1\right) \Phi(h)+h \varphi(h)\right]+\int_{0}^{\infty} \Phi^{2}(h-y) \varphi(h+y) d y \\
& -2 \varphi(h) \Phi(h)[h \Phi(h)+\varphi(h)]-\frac{1}{\sqrt{2}} \int_{0}^{\infty} \Phi(h-y) \varphi(\sqrt{2} h)[\Phi(\sqrt{2} y)-1 / 2] d y .
\end{aligned}
$$

This expression can be approximated as shown in Appendix; see 4.2). 


\subsection{An alternative representation of the Shepp's formula 2.5}

Let $T=n$ be a positive integer, $y_{0}=0, y_{1}=h-x$. For $i=0,1, \ldots, n$ we set $s_{i}=h+y_{i}-y_{i+1}$ with $s_{0}=x$. It follows from Shepp's proof of (2.5) that $s_{0}, s_{1}, \ldots, s_{n}$ have the meaning of the values of the process $S(t)$ at the times $t=0,1, \ldots, n: S(i)=s_{i}(i=0,1, \ldots, n)$. The range of the variables $s_{i}$ is $(-\infty, h)$. The variables $y_{1}, \ldots, y_{n+1}$ are expressed via $s_{0}, \ldots, s_{n}$ by $y_{k}=k h-s_{0}-s_{1}-\ldots-s_{k-1}$ $(k=1, \ldots, n+1)$ with $y_{0}=0$. Changing the variables in $(2.5)$, we obtain

$$
F_{n}(h \mid x)=\frac{1}{\varphi(x)} \int_{-\infty}^{h} \ldots \int_{-\infty}^{h} \operatorname{det} \mid \varphi\left(s_{i}+a_{i, j}\right) \|_{i, j=0}^{n} d s_{1} \ldots d s_{n},
$$

where

$$
a_{i, j}=y_{i+1}-y_{j+1}=\left\{\begin{array}{cc}
0 & \text { for } i=j \\
(i-j) h-s_{j+1}-\ldots-s_{i+1} & \text { for } i>j \\
(i-j) h+s_{i+1}+\ldots+s_{j} & \text { for } i<j
\end{array}\right.
$$

Expression 2.9 for the probability $F_{n}(h \mid x)$ implies that the function

$$
p\left(s_{0}, s_{1}, \ldots s_{n}\right)=\frac{1}{\varphi\left(s_{0}\right) F_{n}\left(h \mid s_{0}\right)} \operatorname{det} \mid \varphi\left(s_{i}+a_{i, j}\right) \|_{i, j=0}^{n} .
$$

is the joint probability density function for the values $S(0), S(1), \ldots, S(n)$ under the condition $S(t)<h$ for all $t \in[0, n]$.

Since $s_{n}$ is the value of $S(n)$, the formula 2.10 also shows the transition density from $s_{0}=x$ to $s_{n}$ conditionally $S(t)<h$ for all $t \in[0, n]$ :

$$
p_{h}^{(n)}\left(x \rightarrow s_{n}\right)=\frac{1}{\varphi(x)} \int_{-\infty}^{h} \ldots \int_{-\infty}^{h} \operatorname{det}\left|\varphi\left(s_{i}+a_{i, j}\right)\right|_{i, j=0}^{n} d s_{1} \ldots d s_{n-1} .
$$

For this transition density, $\int_{-\infty}^{h} p_{h}^{(n)}(x \rightarrow z) d z=F_{n}(h \mid x)$.

\subsection{Approximating $\lambda(h)$ through eigenvalues of integral operators}

\subsubsection{One-step transition}

In the case $n=1$ we obtain from 2.11):

$$
p_{h}^{(1)}(x \rightarrow z)=\frac{1}{\varphi(x)} \operatorname{det}\left(\begin{array}{cc}
\varphi(x) & \varphi(x-h+z) \\
\varphi(h) & \varphi(z)
\end{array}\right)=\varphi(z)\left[1-e^{-(h-z)(h-x)}\right]
$$

with $z=s_{1}<h$.

Let $\lambda_{1}(h)$ be the largest eigenvalue of the the integral operator with kernel 2.12):

$$
\lambda_{1}(h) p(z)=\int_{-\infty}^{h} p(x) p_{h}^{(1)}(x \rightarrow z) d x, z<h,
$$

where eigenfunction $p(x)$ is some probability density on $(-\infty, h]$. The Ruelle-Krasnoselskii-PerronFrobenius theory of bounded linear positive operators (see e.g. Theorem XIII.43 in [13) implies that the maximum eigenvalue $\lambda$ of the operator with kernel $K(x, z)=p_{h}^{(1)}(x \rightarrow z)$ is simple, real and positive and the eigenfunction $p(x)$ can be chosen as a probability density.

Similarly to what we have done below in Section 2.4.2 we can suggest computing good numerical approximations to $\lambda_{1}(h)$ using Gauss-Legendre quadrature formulas. However, we suggest to use (4.15) from [14] instead; this helps us to obtain the following simple but rather accurate approximation to $\lambda_{1}(h)$ :

$$
\hat{\lambda}_{1}(h)=\Phi(h)+\varphi(h) / h-\varphi(h)[\varphi(h)+h \Phi(h)] t /\left[\Phi(h)-e^{-h^{2} / 2} / 2\right] .
$$


Approximation 1: $F_{T}(h) \simeq F_{1}(h)\left[\hat{\lambda}_{1}(h)\right]^{T-1}(T \geq 1) ; \Lambda^{(1)}(h)=-\log \hat{\lambda}_{1}(h), \lambda^{(1)}(h)=\hat{\lambda}_{1}(h)$.

\subsubsection{Transition in a twice longer interval}

Consider now the interval [0,2]. We could have extended the method of Section 2.4.1 and used the eigenvalue (square root of it) for the transition $s_{0} \rightarrow s_{2}$ with transition density expressed in (2.11) with $n=2$. This would improve Approximation 1 but this improvement is only marginal. Instead, we will use another approach: we consider the transition $s_{1} \rightarrow s_{2}$ but use the interval $[0,1]$ just for setting up the initial condition for observing $S(t)$ at $t \in[1,2]$.

For $n=2$, the expression 2.10 for the joint probability density function for the values $S(0), S(1), S(2)$ under the condition $S(t)<h$ for all $t \in[0,2]$ has the form

$$
p\left(s_{0}, s_{1}, s_{2}\right)=\frac{1}{\varphi\left(s_{0}\right) F_{2}\left(h \mid s_{0}\right)} \operatorname{det}\left(\begin{array}{ccc}
\varphi\left(s_{0}\right) & \varphi\left(s_{0}-h+s_{1}\right) & \varphi\left(s_{0}-2 h+s_{1}+s_{2}\right) \\
\varphi(h) & \varphi\left(s_{1}\right) & \varphi\left(s_{1}+s_{2}-h\right) \\
\varphi\left(2 h-s_{1}\right) & \varphi(h) & \varphi\left(s_{2}\right)
\end{array}\right) .
$$

Denote by $p_{1}(z), z<h$, the 'non-normalized' density of $S(1)$ under the condition $S(t)<h$ for all $t \in[0,1]$ that satisfies $\int_{-\infty}^{h} p_{1}(z) d z=F_{1}(h)$. Using 2.12, we obtain

$$
p_{1}(z)=\int_{-\infty}^{h} p_{h}^{(1)}(x \rightarrow z) \varphi(x) d x=\Phi(h) \varphi(z)-\Phi(z) \varphi(h) .
$$

Then the transition density from $x=s_{1}$ to $z=s_{2}$ under the condition $S(t)<h$ for all $t \in[0,2]$ is achieved by integrating $s_{0}$ out and renormalising the joint density:

$$
\begin{aligned}
q_{h}(x \rightarrow z) & =\frac{1}{p_{1}(x)} \int_{-\infty}^{h} \operatorname{det}\left(\begin{array}{ccc}
\varphi\left(s_{0}\right) & \varphi\left(s_{0}-h+x\right) & \varphi\left(s_{0}-2 h+x+z\right) \\
\varphi(h) & \varphi(x) & \varphi(x+z-h) \\
\varphi(2 h-x) & \varphi(h) & \varphi(z)
\end{array}\right) d s_{0} \\
& =\frac{1}{\Phi(h) \varphi(x)-\Phi(x) \varphi(h)} \operatorname{det}\left(\begin{array}{ccc}
\Phi(h) & \Phi(x) & \Phi(x+z-h) \\
\varphi(h) & \varphi(x) & \varphi(x+z-h) \\
\varphi(2 h-x) & \varphi(h) & \varphi(z)
\end{array}\right) .
\end{aligned}
$$

Let $\lambda_{2}(h)$ be the largest eigenvalue of the integral operator with kernel $q_{h}$ :

$$
\lambda_{2}(h) q(z)=\int_{-\infty}^{h} q(x) q_{h}(x \rightarrow z) d x, z<h,
$$

where eigenfunction $q(x)$ is some probability density on $(-\infty, h]$. Similarly to the case $n=1, \lambda_{2}(h)$ is simple, real and positive eigenvalue of the operator with kernel $K(x, z)=q_{h}(x \rightarrow z)$ and the eigenfunction $q(x)$ can be chosen as a probability density.

In numerical examples below we approximate $\lambda_{2}(h)$ using the methodology described in [15, p.154. It is based on the Gauss-Legendre discretization of the interval $[-c, h]$, with some large $c>0$, into an $N$-point set $x_{1}, \ldots, x_{N}$ (the $x_{i}$ 's are the roots of the $N$-th Legendre polynomial on $[-c, h]$ ), and the use of the Gauss-Legendre weights $w_{i}$ associated with points $x_{i} ; \lambda_{2}(h)$ and $q(x)$ are then approximated by the largest eigenvalue and associated eigenvector of the matrix $D^{1 / 2} A D^{1 / 2}$, where $D=\operatorname{diag}\left(w_{i}\right)$, and $A_{i, j}=q_{h}\left(x_{i} \rightarrow x_{j}\right)$. If $N$ is large enough then the resulting approximation $\hat{\lambda}_{2}(h)$ to $\lambda_{2}(h)$ is arbitrarily accurate.

Approximation 2: $F_{T}(h) \simeq F_{2}(h)\left[\hat{\lambda}_{2}(h)\right]^{T-2}(T \geq 2) ; \Lambda^{(2)}(h)=-\log \hat{\lambda}_{2}(h), \lambda^{(2)}(h)=\hat{\lambda}_{2}(h)$.

\subsubsection{Quality of Approximations 1 and 2}

Approximation 1 is more accurate than Approximation 0 but it is still not accurate enough. This is related to the fact that the process $S(t)$ is not Markovian and the behaviour of $S(t)$ on the interval 
$[i, i+1]$ depends on all values of $S(t)$ in the interval $[i-1, i]$ and not only on the value $s_{i}=S(i)$, which is a simplification we used for derivation of Approximation 1. Approximation 2 corrects the bias of Approximation 1 by considering twice longer intervals $[i-1, i+1]$ and using the behaviour of $S(t)$ in the first half of the interval $[i-1, i+1]$ just for setting up the initial condition at $[i, i+1]$. As shown in Section 2.7. Approximation 2 is much more accurate than Approximations 0 and 1. The approximations developed in the following section also carefully consider the dependence of $S(t)$ on its past; they could be made arbitrarily accurate (on expense of increased computational complexity).

\subsection{Main approximations}

As mentioned above, the behaviour of $S(t)$ on the interval $[i, i+1]$ depends on all values of $S(t)$ in the interval $[i-1, i]$ and not only on the value $s_{i}=S(i)$. The exact value of the Shepp's constant $\lambda(h)$ can be defined as the limit (as $i \rightarrow \infty$ ) of the probability that $S(t)<h$ for all $t \in[i, i+1]$ under the condition $S(t)<h$ for all $t \leq i$. Using the formula for conditional probability, we obtain

$$
\lambda(h)=\lim _{i \rightarrow \infty} F_{i}(h) / F_{i-1}(h) .
$$

Waiting a long time without reaching $h$ is not numerically possible and is not what is really required for computation of $\lambda(h)$. What we need is for the process $S(t)$ to (approximately) reach the stationary behaviour in the interval $[i-1, i]$ under the condition $S(t)<h$ for all $t<i$. Since the memory of $S(t)$ is short (it follows from the representation $S(t)=W(t)-W(t+1)$, where $W(t)$ is the standard Wiener process), this stationary behaviour of $S(t)$ is practically achieved for very small $i$, as is seen from numerical results of Section 2.7. Moreover, since ratios $F_{i}(h) / F_{i-1}(h)$ are very close to $F_{i}\left(h \mid x_{h}\right) / F_{i-1}\left(h \mid x_{h}\right)$ for $i \geq 2$, we can use ratios $F_{i}\left(h \mid x_{h}\right) / F_{i-1}\left(h \mid x_{h}\right)$ in 2.13$)$ instead. Here $x_{h}=-\varphi(h) / \Phi(h)$ is the mean of the truncated normal distribution with density $\varphi(x) / \Phi(h)$, $x \leq h$. For computing the approximations, it makes integration easier. Note also another way of justifying the approximation $\lambda(h) \simeq F_{i}(h) / F_{i-1}(h)$ : divide 1.5 with $T=i$ by 1.5 with $T=i-1$.

The above considerations give rise to several approximations formulated below. We start with simpler approximations which are easy to compute and end up with approximations which are extremely accurate but are harder to compute. Approximation 7 is very precise, see Table 3 . However, we would not recommend extremely accurate Approximations 6 and 7 since Approximations 4 and 5 are already very accurate, see Tables 1 and 2, but are much easier to compute. Approximation 3, the simplest in the family, is also quite accurate. Note that all approximations for $F_{T}(h)$ can be applied for any $T \geq 2$.

Approximation 3: $F_{T}(h) \simeq F_{2}(h)\left[\lambda^{(3)}(h)\right]^{T-2}$, where $\lambda^{(3)}(h)=F_{2}\left(h \mid x_{h}\right) / F_{1}\left(h \mid x_{h}\right)$.

Approximation 4: $F_{T}(h) \simeq F_{2}(h)\left[\lambda^{(4)}(h)\right]^{T-2}$, where $\lambda^{(4)}(h)=F_{2}(h) / F_{1}(h)$.

Approximation 5: $F_{T}(h) \simeq F_{2}(h)\left[\lambda^{(5)}(h)\right]^{T-2}$, where $\lambda^{(5)}(h)=F_{3}\left(h \mid x_{h}\right) / F_{2}\left(h \mid x_{h}\right)$.

Approximation 6: $F_{T}(h) \simeq F_{3}(h)\left[\lambda^{(6)}(h)\right]^{T-3}$, where $\lambda^{(6)}(h)=F_{4}\left(h \mid x_{h}\right) / F_{3}\left(h \mid x_{h}\right)$.

Approximation 7: $F_{T}(h) \simeq F_{4}(h)\left[\lambda^{(7)}(h)\right]^{T-4}$, where $\lambda^{(7)}(h)=F_{4}(h) / F_{3}(h)$.

Numerical complexity of these approximation is related to the necessity of computing either $F_{n}(h \mid 0)$ or $F_{n}(h)$ for suitable $n$. It follows from 2.9 that $F_{n}(h \mid 0)$ is an $n$-dimensional integral. Consequently, $F_{n}(h)$ is an $(n+1)$-dimensional integral. In both cases, the dimensionality of the integral can be reduced by one, respectively to $n-1$ and $n$, with no further analytical reduction possible. In view of results of Sections 4.1 and 4.2. computation of Approximations 3 and 4 is easy, computation of Approximation 5 requires numerical evaluation of a one-dimensional integral (which is not hard) but to compute Approximation 7 we need to approximate a three-dimensional integral, 
which has to be done with high precision as otherwise Approximation 7 is not worth using: indeed, Approximations 4-6 are almost as good but are much easier to compute. As Approximation 7 provides us with the values which are practically indistinguishable from the true values of $\lambda(h)$, we use Approximation 7 only for the assessment of the accuracy of other approximations and do not recommend using it in practice.

\subsection{Consistency of approximations when $h$ is large}

Assume that $h \rightarrow \infty$. We shall show that Approximations 3-7 for $\Lambda(h)$ give consistent results with Approximation 0 which is $\Lambda^{(0)}(h)=h \varphi(h)$.

Roughly, this consistency follows if we simply use $\Lambda^{(0)}(h)$ for $\Lambda(h)$ in 1.5$)$ and then substitute the asymptotically correct values of $F_{i-1}(h)$ and $F_{i}(h)$ in $\Lambda(h) \simeq \log F_{i-1}(h)-\log F_{i}(h)$. Similar argument works in the case $\Lambda(h) \simeq \log F_{i-1}\left(h \mid x_{h}\right)-\log F_{i}\left(h \mid x_{h}\right)$.

Consider now Approximation 4 for $\Lambda(h)$, which is $\Lambda^{(4)}(h)=\log F_{1}(h)-\log F_{2}(h)$. From explicit formulas (2.7) and 2.8 for $F_{1}(h)$ and $F_{2}(h)$ we obtain

$$
\begin{aligned}
& F_{1}(h)=1-\left(h+\frac{2}{h}+O\left(\frac{1}{h^{3}}\right)\right) \varphi(h), \quad h \rightarrow \infty, \\
& F_{2}(h)=1-\left(2 h-4-\frac{2}{h}+O\left(\frac{1}{h^{2}}\right)\right) \varphi(h), \quad h \rightarrow \infty,
\end{aligned}
$$

Expansion (2.14) of (2.7) is straightforward. To obtain 2.15) from (2.8) we observe as $h \rightarrow \infty$ :

$$
\Phi^{3}(h)=1-\left(\frac{3}{h}+O\left(\frac{1}{h^{3}}\right)\right) \varphi(h) ; \quad 2 h \varphi(h) \Phi^{2}(h)=\left(2 h-4+O\left(\frac{1}{h^{2}}\right)\right) \varphi(h)
$$

and

$$
\int_{-\infty}^{h} \Phi(y)^{2} \varphi(2 h-y) d y=\left(\frac{1}{h}+O\left(\frac{1}{h^{2}}\right)\right) \varphi(h) ;
$$

all other terms in 2.8 converge to zero (as $h \rightarrow \infty$ ) faster than $\varphi(h) / h^{2}$. Using the expansion $\log (1-x)=-x+O\left(x^{-2}\right)$ as $x \rightarrow 0$, this gives

$$
\Lambda^{(4)}(h)=\log F_{1}(h)-\log F_{2}(h)=\left(h-4-\frac{4}{h}+O\left(\frac{1}{h^{2}}\right)\right) \varphi(h) \text { as } h \rightarrow \infty .
$$

This is fully consistent with approximation $\Lambda^{(0)}(h)$ and all the discussion of Section 2.1. However, there is no guarantee that the constant 4 above is the correct constant in the asymptotic relation

$$
\Lambda(h)=\left(h-\text { const }+O\left(\frac{1}{h}\right)\right) \varphi(h) \text { as } h \rightarrow \infty
$$

provided this asymptotic relation holds.

\subsection{Numerical results}

In this section we discuss the quality of approximations introduced in Section 2 . In Table 1 . we present the values of $\lambda^{(i)}(h), i=0,1, \ldots, 7$, for a number of different $h$; see also Table 3 in Appendix. As mentioned above, $\lambda^{(7)}(h)$ is practically the true $\lambda(h)$ and therefore we compare all other approximations against $\lambda^{(7)}(h)$. In Table 2 we present the relative errors of all other approximations against $\lambda^{(7)}(h)$; that is, the values $\lambda^{(i)}(h) / \lambda^{(7)}(h)-1$ for $i=0,1, \ldots, 6$. From these two tables we see that Approximations 2-7 are very accurate. Moreover, we have made large-scale simulation studies where we have estimated values of $F_{T}(h)$ for different $h$ using $10^{6}$ trajectories of $S(t)$ and all approximations for $F_{T}(h)$ considered above. Visually, Approximations 5-7 are virtually exact (the approximations are always well inside the confidence bounds computed from the simulations) for all $h \geq 0$ and also Approximations 2-4 are visually undistinguishable from them for $h \geq 0.5$. We do not provide corresponding plots as these plots are not informative. 


\begin{tabular}{|c||c|c|c|c|c|c|c|c|c|}
\hline & $h=0$ & $h=0.5$ & $h=1$ & $h=1.5$ & $h=2$ & $h=2.5$ & $h=3$ & $h=3.5$ & $h=4$ \\
\hline$\lambda^{(0)}(h)$ & 1.000000 & 0.838591 & 0.785079 & 0.823430 & 0.897644 & 0.957126 & 0.986792 & 0.996950 & 0.999465 \\
$\lambda^{(1)}(h)$ & 0.250054 & 0.413754 & 0.596156 & 0.762590 & 0.885025 & 0.955674 & 0.986738 & 0.996958 & 0.999466 \\
$\lambda^{(2)}(h)$ & 0.201909 & 0.366973 & 0.563246 & 0.746457 & 0.879719 & 0.954522 & 0.986566 & 0.996939 & 0.999464 \\
$\lambda^{(3)}(h)$ & 0.199421 & 0.366664 & 0.564851 & 0.747979 & 0.880220 & 0.954529 & 0.986532 & 0.996930 & 0.999463 \\
$\lambda^{(4)}(h)$ & 0.200045 & 0.365730 & 0.562888 & 0.746559 & 0.879831 & 0.954556 & 0.986570 & 0.996939 & 0.999464 \\
$\lambda^{(5)}(h)$ & 0.202269 & 0.368099 & 0.564446 & 0.747143 & 0.879943 & 0.954564 & 0.986571 & 0.996939 & 0.999464 \\
$\lambda^{(6)}(h)$ & 0.202455 & 0.368100 & 0.564377 & 0.747118 & 0.879945 & 0.954566 & 0.986571 & 0.996939 & 0.999464 \\
$\lambda^{(7)}(h)$ & 0.202434 & 0.368082 & 0.564371 & 0.747118 & 0.879945 & 0.954566 & 0.986571 & 0.996939 & 0.999464 \\
\hline
\end{tabular}

Table 1: $\lambda^{(i)}(h), i=0,1, \ldots, 7$, for different $h$.

\begin{tabular}{|c|c|c|c|c|c|c|c|c|c|}
\hline & $h=0$ & $h=0.5$ & $h=1$ & $h=1.5$ & $h=2$ & $h=2.5$ & $h=3$ & $h=3.5$ & $h=4$ \\
\hline$\lambda^{(0)}(h)$ & $3.94 \mathrm{e}+00$ & $1.28 \mathrm{e}+00$ & $3.91 \mathrm{e}-01$ & $1.02 \mathrm{e}-01$ & $2.01 \mathrm{e}-02$ & $2.68 \mathrm{e}-03$ & $2.25 \mathrm{e}-04$ & $1.16 \mathrm{e}-05$ & $6.12 \mathrm{e}-07$ \\
\hline$\lambda^{(1)}(h)$ & $2.35 \mathrm{e}-01$ & $1.24 \mathrm{e}-01$ & $5.63 \mathrm{e}-02$ & $2.07 \mathrm{e}-02$ & $5.77 \mathrm{e}-03$ & $1.16 \mathrm{e}-03$ & $1.69 \mathrm{e}-04$ & $1.93 \mathrm{e}-05$ & $1.88 \mathrm{e}-06$ \\
\hline$\lambda^{(2)}(h)$ & $-2.59 \mathrm{e}-03$ & $-3.01 \mathrm{e}-03$ & $-1.99 \mathrm{e}-03$ & $-8.84 \mathrm{e}-04$ & $-2.57 \mathrm{e}-04$ & $-4.56 \mathrm{e}-05$ & $-4.61 e-06$ & $-2.56 \mathrm{e}-07$ & $-7.82 \mathrm{e}-09$ \\
\hline$\lambda^{(3)}(h)$ & $-1.49 \mathrm{e}-02$ & $-3.85 e-03$ & $8.51 \mathrm{e}-04$ & $1.15 \mathrm{e}-03$ & $3.12 \mathrm{e}-04$ & $-3.84 \mathrm{e}-05$ & $-3.88 \mathrm{e}-05$ & $-9.36 e-06$ & $-1.28 \mathrm{e}-06$ \\
\hline$\lambda^{(4)}(h)$ & $-1.18 \mathrm{e}-02$ & $-6.39 e-03$ & $-2.63 e-03$ & $-7.48 \mathrm{e}-04$ & $-1.29 \mathrm{e}-04$ & $-1.09 \mathrm{e}-05$ & $-2.06 e-07$ & $2.27 \mathrm{e}-08$ & $1.35 \mathrm{e}-09$ \\
\hline$\lambda^{(5)}(h)$ & $-8.13 e-04$ & $4.71 \mathrm{e}-05$ & $1.33 \mathrm{e}-04$ & $3.32 \mathrm{e}-05$ & $-2.49 \mathrm{e}-06$ & $-1.57 \mathrm{e}-06$ & $-1.34 \mathrm{e}-07$ & $-2.20 \mathrm{e}-09$ & $9.09 \mathrm{e}-11$ \\
\hline$\lambda^{(6)}(h)$ & $1.03 \mathrm{e}-04$ & $5.02 \mathrm{e}-05$ & $1.09 \mathrm{e}-05$ & $1.88 \mathrm{e}-07$ & $-1.83 e-07$ & $-3.22 \mathrm{e}-11$ & $4.12 \mathrm{e}-11$ & $2.86 \mathrm{e}-11$ & $6.09 \mathrm{e}-12$ \\
\hline
\end{tabular}

Table 2: Relative errors of $\lambda^{(i)}(h), i=0,1, \ldots, 6$, against $\lambda^{(7)}(h)$.

A plot of the relative errors can be seen in Figure 2a, where the number next to the line corresponds to the approximation. Approximations 2,4 and 7 suggest very accurate lower bounds for the true $\lambda(h)$. Approximations 0 and 1 appear to provide upper bounds for $\lambda(h)$ for all $h$.

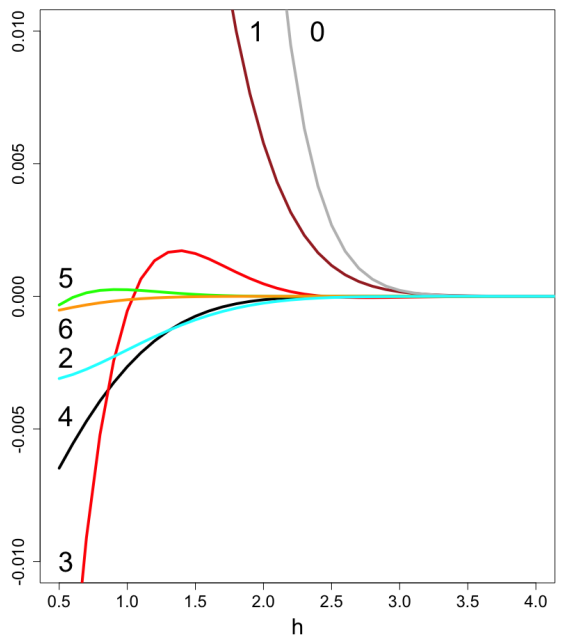

(a) Relative errors of $\lambda^{(i)}(h), i=0, \ldots, 6$, against $\lambda^{(7)}(h)$

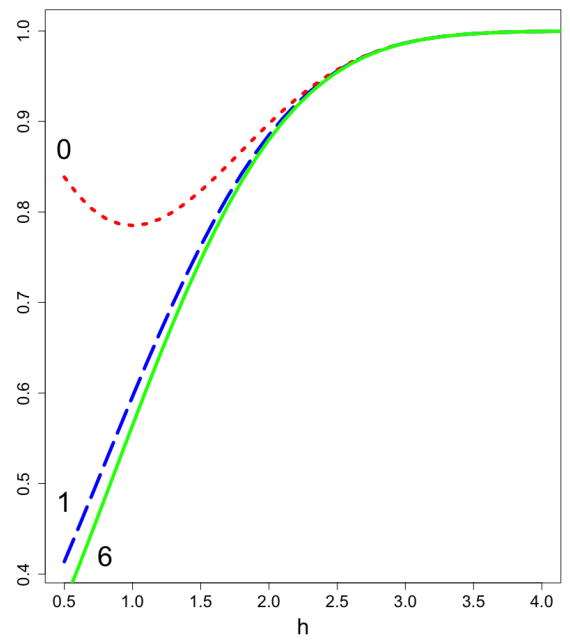

(b) $\lambda^{(0)}(h)$ (dotted red), $\lambda^{(1)}(h)$ (dashed blue) and $\lambda^{(6)}(h)$ (solid green)

Figure 2: Approximations and their relative errors as functions of $h$.

As mentioned in Section 2.4.3. Approximation 1 is not as accurate as Approximations 2-7 because it does not adequately take into account the non-Markovianity of $S(t)$. In Figure 2b we have plotted $\lambda^{(0)}(h)$ (dotted red line), $\lambda^{(1)}(h)$ (dashed red line) and $\lambda^{(6)}(h)$ (solid green line) for a range of interesting $h$. Visually, all $\lambda^{(i)}(h)$ with $i=2,4,5,6,7$ would be visually indistinguishable from each other on the plot in Figure $2 \mathrm{~b}$ and $\lambda^{(3)}(h)$ would be very close to them. The number next to the line corresponds to which approximation was used. 


\section{Comparison of the upper tail asymptotics for the Slepian process against some other stationary Gaussian processes}

Consider the following three stationary Gaussian processes.

1. $\xi_{1}(t)(t \geq 0)$ is the Ornstein-Uhlenbeck process with mean 0 , variance 1 and correlation function $\rho_{1}(t)=\exp (-|t|)$.

2. Let $a>0$ be fixed real number and set $\alpha=\left(1+a+a^{2}\right) /\left(2+2 a+a^{2}\right)$. Then, if $W(t)$ denotes the standard Wiener process, we define the process $\xi_{2}(t)(t \geq 0)$ as follows:

$$
\xi_{2}(t)=\frac{1}{\sqrt{1+a+a^{2}}}\{(1+a) W(t+2 \alpha)-a W(t+\alpha)-W(t)\} .
$$

The process $\xi_{2}(t)$ has mean 0 , variance 1 and correlation function

$$
\rho_{2}(t)= \begin{cases}1-|t|, & \text { for } 0 \leq|t| \leq \alpha \\ \frac{(1+a)(2 \alpha-|t|)}{1+a+a^{2}}, & \text { for } \alpha \leq|t| \leq 2 \alpha \\ 0 & \text { for }|t| \geq 2 \alpha\end{cases}
$$

3. Let $c \geq 1$ be a fixed real number and set $\beta=1 /(c+2)$. Define the process $\xi_{3}(t)$ by

$$
\xi_{3}(t)=\frac{1}{\sqrt{1+c^{2}}}\{W(t+1)+c W(t+(c+1) \beta)-c W(t+\beta)-W(t)\} .
$$

The process $\xi_{3}(t)$ has mean 0 , variance 1 and correlation function

$$
\rho_{3}(t)= \begin{cases}1-|t|, & \text { for } 0 \leq|t| \leq \beta \\ \frac{(1+c)\left(1+c^{2} \beta-|t|(1+c)\right)}{1+c^{2}} & \text { for } \beta \leq|t| \leq c \beta \\ \frac{1+c+c^{2} \beta-|t|(1+2 c)}{1+c^{2}} & \text { for } c \beta \leq|t| \leq(c+1) \beta, \\ \frac{1-|t|}{1+c^{2}}, & \text { for }(c+1) \beta \leq|t| \leq 1 \\ 0, & \text { for }|t| \geq 1 .\end{cases}
$$

It follows from [12, Theorem 3], the above three processes provide a very good representation of the entire class of conditionally Markov stationary Gaussian processes. Indeed, there is only one process in this class where $\alpha \neq 1$ in 2.2 (this is the process with covariance function $\rho(t)=\cos \omega t$ with $\omega \neq 0$ ) and the three types of processes we consider cover well the case where $\alpha=1$ and $C=1$ in 2.2 (the case $C \neq 1$ reduces to the case $C=1$ by substituting $h / C$ for $h$ ). For a graphical representation of the chosen covariance functions, see Figure $3 \mathrm{~b}$

Below we compare Shepp's constant $\Lambda(h)$ defined in 1.4 to similar quantities of the processes $\left\{\xi_{i}(t)\right\},(i=1,2,3)$ defined above. More precisely, let

$$
F_{T, i}(h):=\operatorname{Pr}\left\{\max _{t \in[0, T]} \xi_{i}(t)<h\right\}, \quad i=1,2,3 .
$$

We are interested in comparing Shepp's constant $\Lambda(h)$ with

$$
\Lambda_{i}(h)=-\lim _{T \rightarrow \infty} \frac{1}{T} \log F_{T, i}(h),
$$

for $i=1,2,3$. Importantly, each process has $\mathbb{E} \xi_{i}(t)=0, \mathbb{E} \xi_{i}^{2}(t)=1$ and correlation function $\rho_{i}(t)=\mathbb{E} \xi_{i}(0) \xi_{i}(t)$ which satisfies $\rho_{i}^{\prime}\left(0^{+}\right)=\left.\frac{d}{d t} \rho_{i}(t)\right|_{t=0^{+}}=-1$, for $i=1,2,3$.

The existence and evaluation of the constant $\Lambda_{1}(h)$ defined in 3.1$)$ for the Ornstein-Uhlenbeck process has been considered in [16], where it was shown that $0<\Lambda_{1}(h)<1$ for all $h>0$, and that $\Lambda_{1}(h)$ is the root of a parabolic cylinder function (defined in [16) closest to zero. It is also shown that $\lim _{h \rightarrow 0^{+}} \Lambda_{1}(h)=1$ and $\lim _{h \rightarrow \infty} \Lambda_{1}(h)=h \varphi(h)$. The existence of the constants $\Lambda_{2}(h)$ and $\Lambda_{3}(h)$ follows from similar arguments for the existence of Shepp's constant $\Lambda(h)$. Moreover, 
the constants are approximated by the same methodology as Shepp's constant, namely:

$$
\lambda_{i}(h)=\exp \left(-\Lambda_{i}(h)\right)=\lim _{j \rightarrow \infty} F_{j, i}(h) / F_{j-1, i}(h) .
$$

The justification why we expect $F_{j, i}(h) / F_{j-1, i}(h)$ (with, say, $j \geq 3$ ) to be a good approximation of $\lambda_{i}(h)$ is related to the property of 'fast loss of memory', which processes $\xi_{2}(t)$ and $\xi_{3}(t)$ possess, as the process $S(t)$ does. In view of the complex structure of $\xi_{2}(t)$ and $\xi_{3}(t)$, the values of $F_{T, 2}(h)$ and $F_{T, 3}(h)$ are evaluated via Monte Carlo simulations. In Figure 3a, we compare $\Lambda_{i}(h)$ with Shepp's constant $\Lambda(h)$ (red solid line). $\Lambda_{1}(h)$ (orange dot-dash line) has been computed as in [16]. $\Lambda_{2}(h)$ (blue dashed line) and $\Lambda_{3}(h)$ (dark green dotted line) have been approximated using (3.2 with $j=3$. For $\Lambda_{2}(h)$ we have taken $a=1$ in the definition of $\xi_{2}(t)$ and for $\Lambda_{3}(h)$ we have taken $c=1$ in the definition of $\xi_{3}(t)$. In Figure 3b, we plot the correlation functions: $\rho(t)$ (red solid line); $\rho_{1}(t)$ (orange dot-dash line); $\rho_{2}(t)$ (blue dashed line); $\rho_{3}(t)$ (dark green dotted line). Note that the results obtained are fully consistent with the celebrated 'Slepian's lemma', a Gaussian comparison inequality, see Lemma 1 in [4. In our terms, Slepian's lemma says that if for two stationary Gaussian processes with non-negative covariance functions $\rho_{1}$ and $\rho_{2}$ we have $\rho_{1}(t) \geq \rho_{2}(t)$ for all $t \geq 0$, then for the corresponding values of $\Lambda(h)$ we have $\Lambda_{1}(h) \leq \Lambda_{2}(h)$, for all $h$.

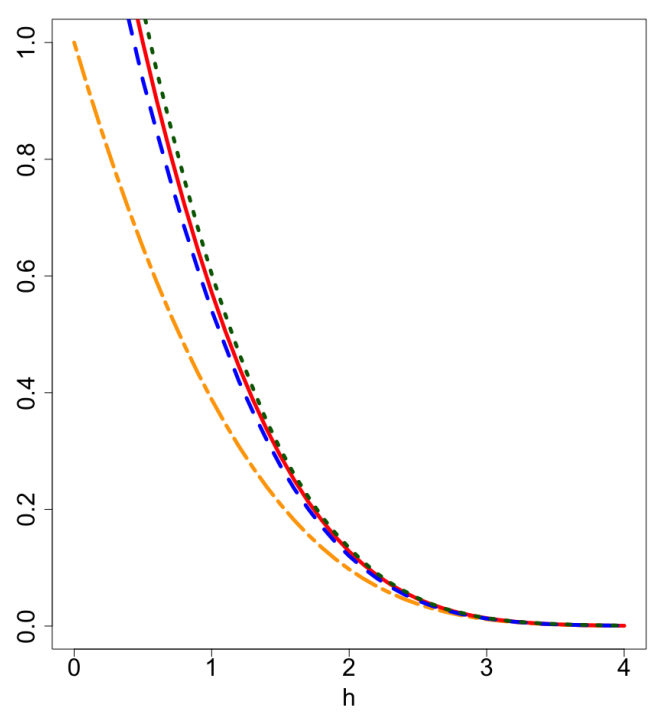

(a) Shepp's constant $\Lambda(h)$ and $\Lambda_{i}(h), i=1,2,3$

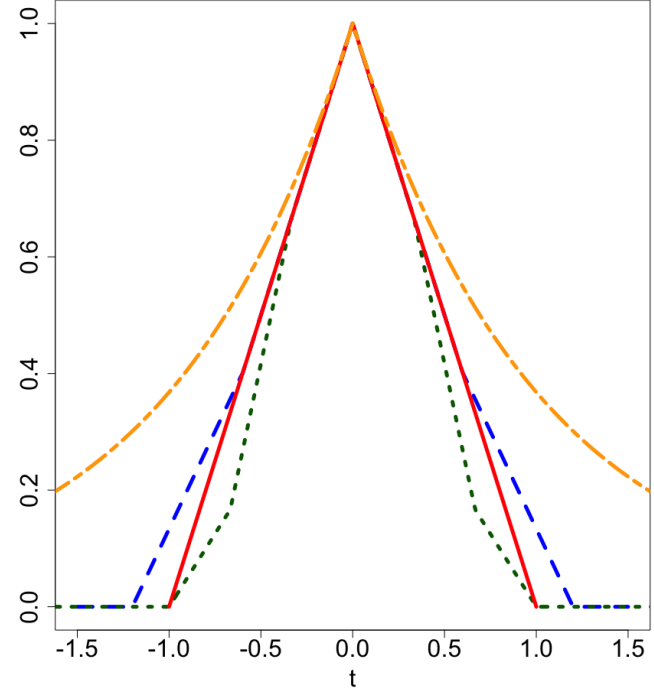

(b) Correlation functions $\rho(t)$ and $\rho_{i}(t), i=1,2,3$

Figure 3: Comparison of the upper tail asymptotics for several Gaussian stationary process

\section{Appendix}

\subsection{Approximations for $\Lambda(h)$}

In Table 3 we use Approximation 7 (our most accurate approximation) to approximate $\Lambda(h)$ over increments 0.1 for $h$. Bold font indicates the decimal places which we claim accurate. Note that $h=0$ has been treated as a special case, see for example [4] and 17]. For $h=0$, instead of Approximation 7, we have used the approximation $\Lambda^{(8)}(h)=-\log \left(F_{5}(h) / F_{4}(h)\right)$; we do not recommend using this approximation in general because of its high complexity. 


\begin{tabular}{|c|c||c|c||c|c||c|c||c|c|}
\hline$h$ & $\Lambda(h)$ & $h$ & $\Lambda(h)$ & $h$ & $\Lambda(h)$ & $h$ & $\Lambda(h)$ & $h$ & $\Lambda(h)$ \\
\hline 0.0 & $\mathbf{1 . 5 9 7 2}$ & 0.8 & $\mathbf{0 . 7 2 4 0}$ & 1.6 & $\mathbf{0 . 2 5 0 5 1 9}$ & 2.4 & $\mathbf{0 . 0 5 7 8 9 4 4}$ & 3.2 & $\mathbf{0 . 0 0 7 7 0 1 6}$ \\
0.1 & $\mathbf{1 . 4 6 3}$ & 0.9 & $\mathbf{0 . 6 4 5 0}$ & 1.7 & $\mathbf{0 . 2 1 3 9 2 9}$ & 2.5 & $\mathbf{0 . 0 4 6 4 9 8 6}$ & 3.3 & $\mathbf{0 . 0 0 5 7 2 4 4}$ \\
0.2 & $\mathbf{1 . 3 3 6 5}$ & 1.0 & $\mathbf{0 . 5 7 2 0}$ & 1.8 & $\mathbf{0 . 1 8 1 4 8 4}$ & 2.6 & $\mathbf{0 . 0 3 7 0 1 2 2}$ & 3.4 & $\mathbf{0 . 0 0 4 2 1 1 1}$ \\
0.3 & $\mathbf{1 . 2 1 7 0}$ & 1.1 & $\mathbf{0 . 5 0 5 1}$ & 1.9 & $\mathbf{0 . 1 5 2 9 0 2}$ & 2.7 & $\mathbf{0 . 0 2 9 1 9 0 9}$ & 3.5 & $\mathbf{0 . 0 0 3 0 6 5 8}$ \\
0.4 & $\mathbf{1 . 1 0 4 7}$ & 1.2 & $\mathbf{0 . 4 4 3 8}$ & 2.0 & $\mathbf{0 . 1 2 7 8 9 6}$ & 2.8 & $\mathbf{0 . 0 2 2 8 0 5 8}$ & 3.6 & $\mathbf{0 . 0 0 2 2 0 8 7}$ \\
0.5 & $\mathbf{0 . 9 9 9 5}$ & 1.3 & $\mathbf{0 . 3 8 7 9}$ & 2.1 & $\mathbf{0 . 1 0 6 1 7 8}$ & 2.9 & $\mathbf{0 . 0 1 7 6 4 6 2}$ & 3.7 & $\mathbf{0 . 0 0 1 5 7 4 7}$ \\
0.6 & $\mathbf{0 . 9 0 1 0}$ & 1.4 & $\mathbf{0 . 3 3 7 2}$ & 2.2 & $\mathbf{0 . 0 8 7 4 6 0}$ & 3.0 & $\mathbf{0 . 0 1 3 5 2 0 3}$ & 3.8 & $\mathbf{0 . 0 0 1 1 1 0 9}$ \\
0.7 & $\mathbf{0 . 8 0 9 2}$ & 1.5 & $\mathbf{0 . 2 9 1 5}$ & 2.3 & $\mathbf{0 . 0 7 1 4 5 8}$ & 3.1 & $\mathbf{0 . 0 1 0 2 5 6 1}$ & 3.9 & $\mathbf{0 . 0 0 0 7 7 5 5}$ \\
\hline
\end{tabular}

Table 3: Approximations for $\Lambda(h)$ with accurate decimal digits in bold.

\subsection{An approximation for $F_{2}(h)$}

Using approximations for $\Phi(t)$, it is possible to approximate $F_{2}(h)$ very accurately. For example, using the approximation (see [18])

$$
\Phi(t)= \begin{cases}0.5 \exp \left(0.717 t-0.416 t^{2}\right) & \text { for } t \leq 0 \\ 1-0.5 \exp \left(-0.717 t-0.416 t^{2}\right) & \text { for } t>0\end{cases}
$$

we obtain

$$
\begin{aligned}
F_{2}(h) & \cong \Phi(h)^{3}+\varphi(h)^{2} \Phi(h)+\frac{\varphi(h)^{2}}{2}\left[\left(h^{2}-1\right) \Phi(h)+h \varphi(h)\right]-2 \varphi(h) \Phi(h)[h \Phi(h)+\varphi(h)] \\
& +\Phi(2 h)-\Phi(h)-\frac{0.5}{\sqrt{2 \pi}} e^{-2 h^{2}}\left[2 J(0.916, b, h)-\frac{1}{2} J\left(1.332, b_{1}, h\right)-\frac{1}{\sqrt{2 \pi}} V(1.416, b, h)\right. \\
& \left.+\frac{2}{\sqrt{2 \pi}} V\left(1, b_{2}, h\right)+\frac{1}{\pi} K\left(1.5, b_{2}, h\right)-\frac{1}{2}\left\{K\left(1.332, b_{3}, 0\right)-\frac{2}{\sqrt{2 \pi}} U\left(1.416, b_{4}, 0\right)\right\}\right],
\end{aligned}
$$

where $b=2 h-0.717, b_{1}=b-0.717, b_{2}=2 h, b_{3}=b+2.151, b_{4}=b+1.434$,

$$
K(x, y, z)=\frac{\sqrt{\pi} e^{y^{2} /(4 x)}}{\sqrt{x}} \Phi\left(\frac{2 x z-y}{\sqrt{2 x}}\right), U(x, y, z)=\frac{1}{2 x}\left[y K(x, y, z)-e^{z(y-x z)}\right],
$$

$J(x, y, z)=K(x, y, z)-K(x, y, 0)$ and $V(x, y, z)=U(x, y, z)-U(x, y, 0)$.

Table 4 shows that approximation 4.2 is very accurate across all $h$ of interest.

\begin{tabular}{|c||c|c|c|c|c|c|c|c|c|}
\hline & $h=0$ & $h=0.5$ & $h=1$ & $h=1.5$ & $h=2$ & $h=2.5$ & $h=3$ & $h=3.5$ & $h=4$ \\
\hline$F_{2}(h)$ & 0.018173 & 0.085014 & 0.250896 & 0.502268 & 0.744845 & 0.900875 & 0.970790 & 0.993430 & 0.998866 \\
$(4.2)$ & 0.019548 & 0.084687 & 0.250203 & 0.502097 & 0.744837 & 0.900875 & 0.970790 & 0.993430 & 0.998866 \\
\hline
\end{tabular}

Table 4: Accuracy of approximation 4.2 for $F_{2}(h)$

\subsection{Simplified form of $F_{2}\left(h \mid x_{h}\right)$ and its approximation}

Using (2.5), for any $x_{0} \leq 0$, we can express $F_{2}\left(h \mid x_{0}\right)$ as follows:

$$
\begin{aligned}
F_{2}\left(h \mid x_{0}\right) & =\Phi(h)^{2}+\frac{1}{\varphi\left(x_{0}\right)} \varphi(h)^{2} x_{0} \Phi\left(x_{0}\right)-\frac{1}{\varphi\left(x_{0}\right)} \varphi(h) \Phi(h) \Phi\left(x_{0}\right)-h \varphi(h) \Phi(h) \\
& +\frac{1}{\varphi\left(x_{0}\right)} \int_{h}^{\infty} \varphi(y) \Phi(2 h-y) \varphi\left(h+x_{0}-y\right) d y-\frac{1}{\varphi\left(x_{0}\right)} \int_{h}^{\infty} \Phi\left(h+x_{0}-y\right) \varphi(2 h-y) \varphi(y) d y .
\end{aligned}
$$

Using (4.1), we obtain the approximation $F_{2}\left(h \mid x_{0}\right) \cong \hat{F}$ where 


$$
\begin{aligned}
\hat{F} & =\Phi(h)^{2}+\frac{1}{\varphi\left(x_{0}\right)} \varphi(h)^{2} x_{0} \Phi\left(x_{0}\right)-\frac{1}{\varphi\left(x_{0}\right)} \varphi(h) \Phi(h) \Phi\left(x_{0}\right)-h \varphi(h) \Phi(h) \\
& +\frac{1}{\sqrt{2} \varphi\left(x_{0}\right)} \varphi\left(\frac{h+x_{0}}{\sqrt{2}}\right)\left[\Phi\left(2 h \sqrt{2}-\frac{h+x_{0}}{\sqrt{2}}\right)-\Phi\left(h \sqrt{2}-\frac{h+x_{0}}{\sqrt{2}}\right)\right] \\
& -\frac{\varphi\left(h+x_{0}\right)}{2 \sqrt{2 \pi} \varphi\left(x_{0}\right)} e^{-1.664 h^{2}}\left\{e^{-1.434 h}\left[K\left(1.416,2.664 h+x_{0}+0.717,2 h\right)-K\left(1.416,2.664 h+x_{0}+0.717, h\right)\right]\right. \\
& \left.-e^{1.434 h}\left[K\left(1.416,2.664 h+x_{0}-0.717, \infty\right)-K\left(1.416,2.664 h+x_{0}-0.717,2 h\right)\right]\right\} \\
& -\frac{e^{0.717\left(h+x_{0}\right)-2 h^{2}-0.416\left(h+x_{0}\right)^{2}}}{2 \varphi\left(x_{0}\right) 2 \pi}\left[K\left(1.416,2 h+0.832\left(h+x_{0}\right)-0.717, \infty\right)\right. \\
& \left.-K\left(1.416,2 h+0.832\left(h+x_{0}\right)-0.717, h\right)\right]
\end{aligned}
$$

where $K(x, y, z)$ is defined in 4.3). Table 5 shows that approximation (4.4) is very accurate for $x_{0}=x_{h}=-\varphi(h) / \Phi(h)$, for any $h \geq 0$.

\begin{tabular}{|c||c|c|c|c|c|c|c|c|c|}
\hline & $h=0$ & $h=0.5$ & $h=1$ & $h=1.5$ & $h=2$ & $h=2.5$ & $h=3$ & $h=3.5$ & $h=4$ \\
\hline$F_{2}\left(h \mid x_{0}\right)$ & 0.041459 & 0.141066 & 0.337112 & 0.588949 & 0.803170 & 0.927924 & 0.979740 & 0.995608 & 0.999264 \\
$\hat{F}$ & 0.041942 & 0.139821 & 0.336115 & 0.588695 & 0.803139 & 0.927922 & 0.979740 & 0.995608 & 0.999264 \\
\hline
\end{tabular}

Table 5: Accuracy of approximation $\hat{F} \simeq F_{2}(h \mid 0)$

\section{Conclusions}

In his seminal paper [1, L. Shepp derived explicit formulas for $F_{T}(h)=\operatorname{Pr}\left\{\max _{t \in[0, T]} S(t)<h\right\}$, the distribution of maximum of the so-called Slepian process $S(t)$. As these explicit formulas are complicated, in the same paper L. Shepp has introduced a constant $\Lambda(h)=-\lim _{T \rightarrow \infty} \frac{1}{T} \log F_{T}(h)$ (which we call Shepp's constant) measuring the rate of decrease of $F_{T}(h)$ as $T$ grows; L. Shepp also raised the question of constructing accurate approximations and bounds for this constant. Until now, this question has not been adequately addressed. To answer it, we have constructed different approximations for $F_{T}(h)$ (and hence for $\Lambda(h)$ ). We have shown in Section 2.7 that at least some of these approximations are extremely accurate for all $h \geq 0$. We have also provided other approximations that are almost as good but are much simpler to compute.

\section{Acknowledgement}

The authors are grateful to the AE and both reviewers whose comments much helped for improving the presentation.

\section{References}

[1] L. Shepp. First passage time for a particular Gaussian process. The Annals of Mathematical Statistics, 42(3):946-951, 1971.

[2] D. Slepian. First passage time for a particular Gaussian process. The Annals of Mathematical Statistics, 32(2):610-612, 1961.

[3] Wenbo V Li and Qi-Man Shao. Lower tail probabilities for Gaussian processes. The Annals of Probability, 32(1):216-242, 2004. 
[4] D. Slepian. The one-sided barrier problem for Gaussian noise. Bell System Technical Journal, 41(2):463-501, 1962.

[5] G. Molchan. Survival exponents for some Gaussian processes. International Journal of Stochastic Analysis, 2012.

[6] H.J. Landau and L.A. Shepp. On the supremum of a Gaussian process. Sankhyā: The Indian Journal of Statistics, Series A, 32:369-378, 1970.

[7] M. Marcus and L.A. Shepp. Sample behavior of Gaussian processes. In Proc. of the Sixth Berkeley Symposium on Math. Statist. and Prob, 2:423-421, 1972.

[8] R.J. Adler and J. Taylor. Random Fields and Geometry. Springer, 2007.

[9] J. Pickands. Upcrossing probabilities for stationary Gaussian processes. Transactions of the American Mathematical Society, 145:51-73, 1969.

[10] A.J. Harper. Pickands constant $H_{\alpha}$ does not equal 1/ $\Gamma(1 / \alpha)$, for small $\alpha$. Bernoulli, 23(1):582$602,2017$.

[11] D. Aldous. Probability Approximations via the Poisson Clumping Heuristic. Springer Science \& Business Media, 1989.

[12] C.B. Mehr and J.A. McFadden. Certain properties of Gaussian processes and their first-passage times. Journal of the Royal Statistical Society. Series B (Methodological), 27(3):505-522, 1965.

[13] M. Reed and B. Simon. Methods of Modern Mathematical Physics: Scattering theory Vol. 3. Academic Press, 1979.

[14] J. Noonan and A. Zhigljavsky. Approximations of the boundary crossing probabilities for the maximum of moving sums. arXiv preprint arXiv:1810.09229, 2018.

[15] J.L. Mohamed and L.M. Delves. Computational Methods for Integral Equations. Cambridge University Press, 1985.

[16] J.A. Beekman. Asymptotic distributions for the Ornstein-Uhlenbeck process. Journal of Applied Probability, 12(1):107-114, 1975.

[17] J. Pitman and W. Tang. The Slepian zero set, and Brownian bridge embedded in Brownian motion by a spacetime shift. Electronic Journal of Probability, 20(61):128, 2015.

[18] J.T. Lin. Approximating the normal tail probability and its inverse for use on a pocket calculator. Applied Statistics, 38(1):69-70, 1989. 